\title{
How does blood glucose control with insulin save lives in intensive care?
}

\author{
Greet Van den Berghe
}

Department of Intensive Care Medicine, Catholic University of Leuven, Leuven, Belgium.

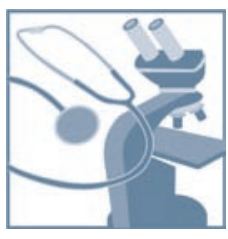

\begin{abstract}
Patients requiring prolonged intensive care are at high risk for multiple organ failure and death. Insulin resistance and hyperglycemia accompany critical illness, and the severity of this "diabetes of stress" reflects the risk of death. Recently it was shown that preventing hyperglycemia with insulin substantially improves outcome of critical illness. This article examines some potential mechanisms underlying prevention of glucose toxicity as well as the effects of insulin independent of glucose control. Unraveling the molecular mechanisms will provide new insights into the pathogenesis of multiple organ failure and open avenues for novel therapeutic strategies.
\end{abstract}

\section{Historical introduction}

The 1952 Scandinavian epidemic of poliomyelitis necessitated mechanical ventilation of a large number of patients with respiratory failure, an intervention that reduced mortality from $80 \%$ to $40 \%$ (1). Since then, development of sophisticated mechanical devices to support all vital organ functions, a wide array of powerful drugs, and high-tech monitoring systems have revolutionized modern intensive-care medicine. This evolution improved short-term survival of previously lethal conditions such as multiple trauma, extensive burns, major surgery, and severe sepsis. Many patients nowadays indeed survive the initial shock phase of such conditions but often subsequently enter a chronic phase of critical illness. Mortality among such patients requiring intensive care for more than a few days has remained around $20 \%$ worldwide, to a large extent irrespective of the initial disease or trauma for which they were admitted to the intensive care unit (ICU). Most deaths in the ICU occurring beyond the first few days of critical illness are attributable to nonresolving failure of multiple organ systems, either due to or coinciding with sepsis. An increased susceptibility to infectious complications and the functional and structural sequelae of the systemic inflammatory response to infection and cellular injury play a role (2). Several lines of evidence support the concept that disturbed cellular energy metabolism contributes to organ failure $(3,4)$. This was originally ascribed exclusively to inadequate tissue perfusion and cellular hypoxia. Recent studies, however, also point to a disturbance in oxygen utilization rather than delivery, which has been termed cytopathic hypoxia $(3,5,6)$. Although extensive research efforts during the last decade focused on strategies to prevent or reverse the potentially lethal multiple organ failure, only few of them revealed positive results $(7-10)$. One of these strategies is tight blood glucose control with insulin (10).

Nonstandard abbreviations used: CRP, C-reactive protein; GABA, $\gamma$-aminobutyric acid; GH, growth hormone; GHBP, GH-binding protein; GIK, glucose, insulin, and potassium; HXK-II, hexokinase II; ICU, intensive care unit; IGFBP-1, IGF-binding protein-1; IRS-1, insulin receptor substrate-1; MnSOD, manganese superoxide dismutase; PEPCK, phosphoenolpyruvate carboxykinase; VDAC, voltage-dependent anion channel.

Conflict of interest: The author holds an unrestrictive Catholic University of Leuven - Novo Nordisk Chair of Research.

Citation for this article: J. Clin. Invest. 114:1187-1195 (2004). doi:10.1172/JCI200423506.
Insulin resistance and hyperglycemia in the critically ill

One hundred fifty years ago, Reyboso observed glucosuria, a condition induced by ether anesthesia, in which glucose is discharged in the urine, and in 1877 Claude Bernard described hyperglycemia during hemorrhagic shock (11). Today, it is well known that any type of acute illness or injury results in insulin resistance, glucose intolerance, and hyperglycemia, a constellation termed "diabetes of injury" $(12,13)$. Illness or trauma increases hepatic glucose production with ongoing gluconeogenesis despite hyperglycemia and abundantly released insulin. Hepatic insulin resistance is further characterized by elevated circulating levels of IGF-binding protein-1 (IGFBP-1) $(14,15)$. Also, in skeletal muscle and heart, insulin-stimulated glucose uptake is impaired $(16,17)$. Overall, glucose uptake in critically ill patients, however, is increased but takes place mainly in the tissues that are not dependent on insulin for glucose uptake, such as, among others, the nervous system and the blood cells $(13,18)$. The most severe cases of stress-induced hyperglycemia (13) and highest levels of circulating IGFBP-1 $(14,15)$ are observed in patients with the highest risk of death. Orchestrated "counterregulatory" hormonal responses, cytokine release, and signals from the nervous system, all affecting glucose metabolic pathways, bring about the diabetes of injury. The hormones involved include catecholamines, cortisol, glucagon, and growth hormone (GH). Proinflammatory cytokines affect glucose homeostasis indirectly, by stimulating counterregulatory hormone secretion, and directly, by altering insulin receptor signaling (Figure 1) $(13,19,20)$. Although insulin receptor signaling is still incompletely understood, generation of SOCS- 1 and SOCS-3 may be involved. Indeed, IL-6-stimulated SOCS-3 generation has been shown to inhibit insulin receptor tyrosine phosphorylation and downstream signal transduction (21), and both SOCS-1 and SOCS-3 have been shown to degrade insulin receptor substrate-1 (IRS-1) and IRS-2 (22). Furthermore, both endogenous and exogenous catecholamines promptly inhibit insulin secretion from $\beta$ cells, and catecholamines as well as angiotensin II exert anti-insulin effects. Abnormalities in insulin signaling have been described in a rat model of critical illness resulting from prolonged administration of a nonlethal dose of endotoxin and concomitant starvation partly mimicking the condition of human sepsis (23). In the liver, abundance and tyrosine phosphorylation of the insulin receptor, IRS-1, and IRS- 2 were reduced. Furthermore, there was reduced association of PI3K with IRS-1. In skeletal muscle, 


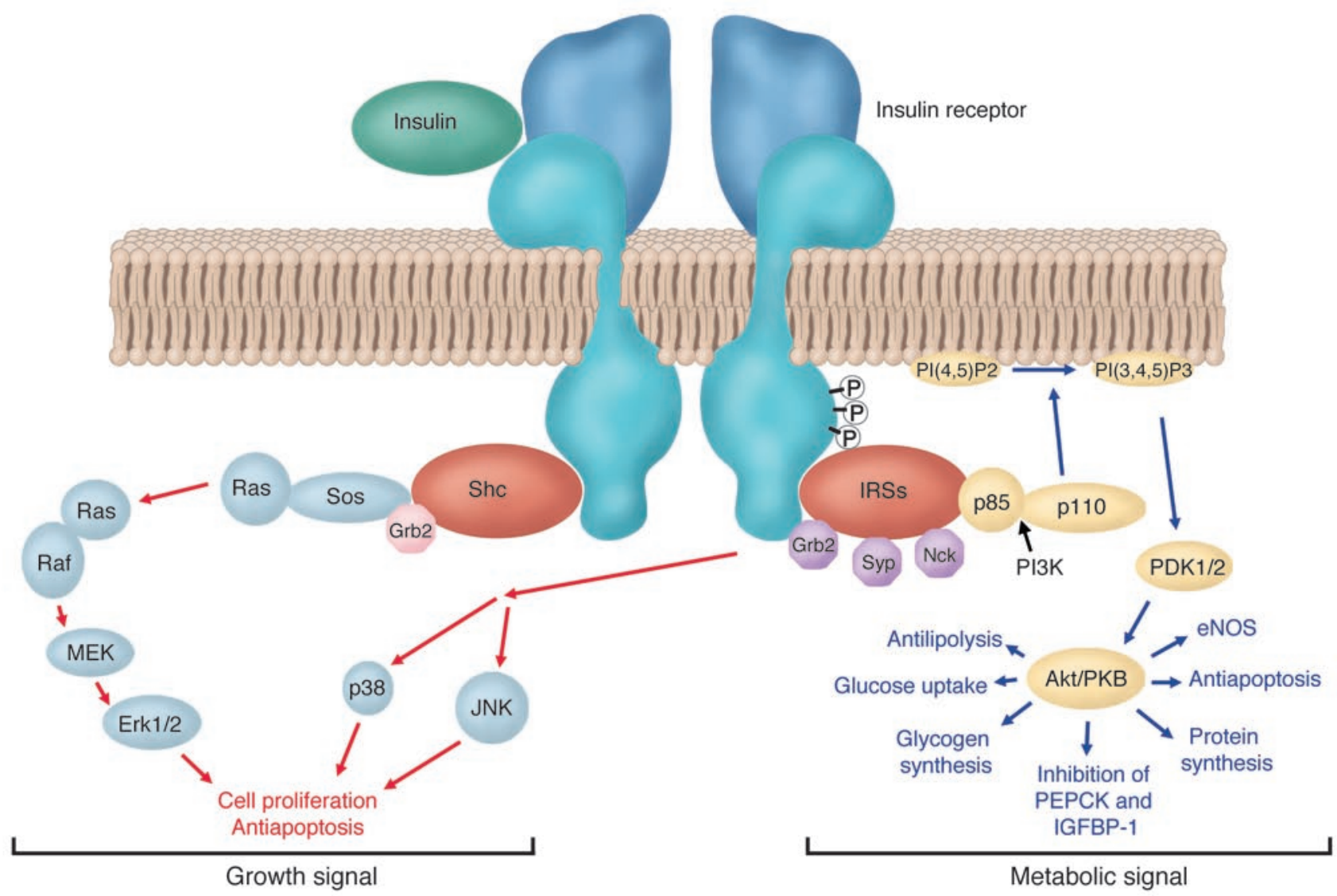

Figure 1

Simplified model of insulin signaling. Insulin binding to the extracellular domain of the insulin receptor elicits a conformational change, which in turn leads to receptor autophosphorylation $(P)$ and tyrosine phosphorylation of intracellular protein substrates. Two main branching pathways are activated by insulin: (a) One is the MAPK signaling cascade, in which the Grb2/Sos pathway leads to activation of Ras signaling, affecting cell proliferation and apoptosis. In view of their mitogenic nature, these can be characterized as "growth signal" effects. (b) The other is the IRS pathway, which leads to activation of kinases dependent upon the heterodimeric (p85/p110) PI3K, such as Akt, also referred to as protein kinase B (PKB); Akt modulates enzyme activities that, besides affecting NO generation and apoptosis, control glucose, lipid, and protein metabolism. This PI3K-branching pathway is termed the "metabolic signal." PI(4,5)P2, phosphoinositide 4,5 di-phosphate; PI(3,4,5)P3, phosphoinositide 3,4,5 tri-phosphate; PDK1 phosphoinositide-dependent kinase-1; MEK, MAPK kinase.

similar abnormalities were observed, except that the number of insulin receptors and the abundance of the IRSs were normal. Furthermore, the insulin signaling defects in the muscle were not seen when rats were studied shortly after injection of endotoxin or under conditions of adequate nutrition (23). Apart from these insights generated from in vitro or animal models, little is known about the exact molecular basis of insulin resistance in critically ill patients. Type 2 diabetes mellitus, and to a lesser extent obesity, are also characterized by hyperglycemia, reduced glucose uptake and oxidation, unsuppressed gluconeogenesis, and impaired glycogen and NO synthesis. Here, the metabolic consequences of insulin resistance are mediated predominantly by abnormalities along the IRS-1-PI3K pathway of insulin signaling (Figure 1). However, a disrupted PI3K pathway does not necessarily mean that the other insulin signaling pathways are equally unresponsive. Indeed, signaling through the Ras-MAPK cascade, for example, via Erk 1 and Erk 2, may retain normal sensitivity. Compensatory hyperinsulinemia may thus still exert mitogenic actions in certain cell types, while the PI3K-dependent metabolic actions of insulin are suppressed (24-28). This discrepancy may occur in vascular smooth muscle cells and in specific capillary endothelial cells of patients with type 2 diabetes and obesity. Proliferation of retinal capillary endothelial cells results in microaneurysms and neovascularization. Excessive proliferation of arterial smooth muscle cells and increased extracellular matrix lead to atherosclerosis. Hence, compensatory hyperinsulinemia, due to metabolic insulin resistance, may contribute to some of the vascular complications of type 2 diabetes and obesity through overstimulation of the mitogenic, insulin-sensitive MAPK signaling pathway. Whether or not insulin resistance is similarly "selective" during critical illness, and whether hyperinsulinemia exerts deleterious effects through this pathway in the acute setting of severe illness, is at present unknown. The diabetes of injury used to be interpreted as an adaptive stress response and as such important for survival. Particularly, the overall increase in glucose turnover and the fact that hyperglycemia persists despite abundantly released insulin were considered arguments in favor of tolerating moderately elevated blood glucose levels during critical illness. Indeed, if one considers hyperglycemia of injury as beneficial in promoting cellular glucose uptake in non-insulin-dependent tissues, tolerating modest degrees of hyperglycemia is beneficial. Consequently, blood glucose concentrations of $160-200 \mathrm{mg} / \mathrm{dl}$ were recommended to max- 
imize cellular glucose uptake while avoiding hyperosmolarity (18). In addition, moderate hyperglycemia was often viewed as a buffer against hypoglycemia-induced brain damage. In 2001, however, the critical care community was forced to reconsider this dogma (29), as a large, randomized, controlled, clinical study - hereafter referred to as "the Leuven study" - showed that preventing even moderate hyperglycemia during critical illness substantially improved outcome (10).

\section{Intensive insulin therapy in critical illness: clinical benefits}

The Leuven study of critically ill patients, the majority of whom did not previously have diabetes, showed that titrating insulin infusion during intensive care to strict normoglycemia (below 110 $\mathrm{mg} / \mathrm{dl}$ ) strikingly reduced mortality when compared with the conventional insulin treatment (Figure 2). The latter comprised insulin infusion only when blood glucose exceeded $200 \mathrm{mg} / \mathrm{dl}$, leading to average blood glucose levels of $150-160 \mathrm{mg} / \mathrm{dl}$ (10). Although the Diabetes Mellitus, Insulin Glucose Infusion in Acute Myocardial Infarction study previously showed that avoiding excessive hyperglycemia (blood glucose $>200 \mathrm{mg} / \mathrm{dl}$ ) after acute myocardial infarction in patients with diabetes mellitus improved long-term outcome (30), the Leuven study (10) on nondiabetic ICU patients aimed for a much lower level of blood glucose. The benefit of intensive insulin therapy in the ICU was particularly apparent among patients with prolonged critical illness, requiring intensive care for more than 5 days, with mortality reduced from $20.2 \%$ to $10.6 \%$. Besides saving lives, intensive insulin therapy also prevented complications such as severe nosocomial infections, acute renal failure, liver dysfunction, critical illness polyneuropathy, muscle weakness, and anemia and thus reduced the time that patients were dependent on intensive care. Although intensive insulin therapy induced a slightly higher incidence of hypoglycemia as compared with the conventional approach, these episodes were never associated with clinically relevant sequelae. Indeed, the use of an insulin titration algorithm guaranteed that when hypoglycemia occurred, it was always quickly resolved. Although the Leuven study included a large number of patients recovering from cardiac surgery, the beneficial effects of strict glucose control were equally present in most other diagnostic subgroups. Furthermore, Krinsley recently confirmed the survival benefit of implementing tight blood glucose control with insulin in a mixed medical-surgical intensive care population (31). The substantial improvement of outcome with such a simple measure was considered major progress in the modern era of intensive care.

\section{Mechanisms explaining the acute life-saving effects of intensive insulin therapy in the ICU}

One immediately wonders how such a simple intervention - preventing a moderate degree of hyperglycemia with insulin - during the relatively short time a patient is in intensive care was able to prevent the most feared complications such as sepsis, multiple organ failure, and death. Normal cells are relatively protected from deleterious effects of brief exposure to moderate hyperglycemia by a downregulation of glucose transporters (32). In diabetes mellitus, prolonged untreated hyperglycemia contributes to the development of chronic debilitating complications. However, except in embryonic development, where hyperglycemia causes acute toxicity, the time required for hyperglycemia to cause disorders in patients with diabetes is several orders of magnitude longer than the time it took to prevent life-threatening complications with insulin therapy in the ICU. In order to understand how metabolic control with insulin proved to be so acutely protective in the critically ill, the following key questions must be answered. Is glycemic control crucial in bringing about the clinical benefits of intensive insulin therapy, or is blood glucose control epiphenomenal to the other metabolic and nonmetabolic effects of insulin? Are there factors predisposing the critically ill to hyperglycemia-induced toxicity? What are the common pathways mediating the plethora of clinical benefits of intensive insulin therapy?

\section{Preventing direct glucose toxicity plays a crucial role}

Clinical evidence. A post hoc analysis of the Leuven study (33) revealed a linear correlation between the degree of hyperglycemia and the risk of death, which persisted after correction for insulin dose and severity of illness scores. Patients in the conventional insulin treatment group who showed only moderate hyperglycemia $(110-150 \mathrm{mg} / \mathrm{dl}$ or $6.1-8.3 \mathrm{mmol} / \mathrm{l}$ ) had a lower risk of death than those with frank hyperglycemia $(150-200 \mathrm{mg} / \mathrm{dl}$ or $>8.3 \mathrm{mmol} / \mathrm{l})$ but a higher risk of death than those who were intensively treated with insulin to restore blood glucose levels to below $110 \mathrm{mg} / \mathrm{dl}$ (6.1 mmol/l) (33). Similarly, for the prevention of morbidity effects such as acute renal failure, bacteremia, and anemia, it appeared crucial to reduce blood glucose to below $110 \mathrm{mg} / \mathrm{dl}$. The risk of developing critical illness polyneuropathy in particular correlated linearly with blood glucose levels (34). Multivariate logistic regression analysis confirmed the independent role of blood glucose control in achieving most of the clinical benefits of intensive insulin therapy and underlined the importance of lowering the blood glucose level to strict normoglycemia.

Target tissue responsiveness to insulin in the critically ill. Since critically ill patients suffer from hepatic and skeletal muscle insulin resistance, the mechanism by which insulin lowered blood glucose in
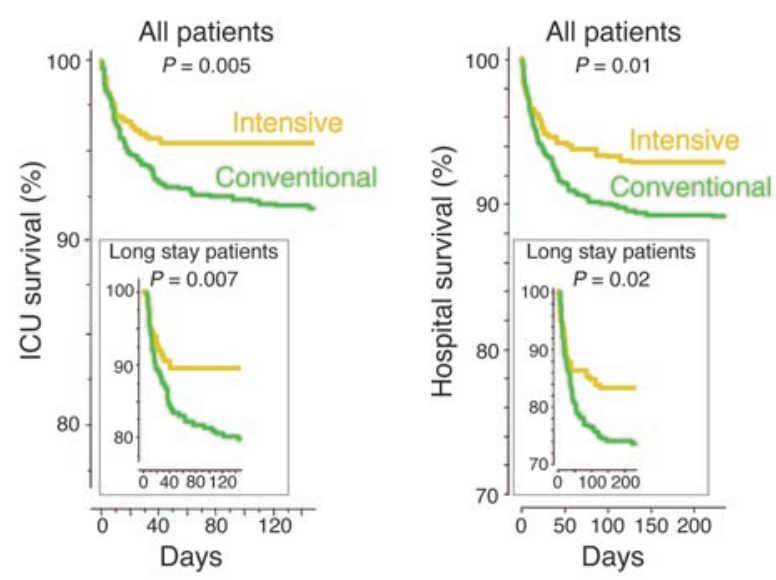

\section{Figure 2}

Intensive insulin therapy saves lives in the intensive care unit. KaplanMeier curves show cumulative survival of 1,548 patients from the Leuven study who received intensive insulin treatment (blood glucose maintained below $110 \mathrm{mg} / \mathrm{dl}$; yellow) or conventional insulin treatment (insulin only given when blood glucose exceeded $200 \mathrm{mg} / \mathrm{dl}$, resulting in mean blood glucose levels of $150-160 \mathrm{mg} / \mathrm{dl}$; green) during their ICU or hospital stay. The upper panels display results from all patients; the lower panels display results for long-stay ( $>5$ days) ICU patients only. $P$ values were determined with the use of the MantelCox log-rank test. Adapted with permission from the New England Journal of Medicine (10). 


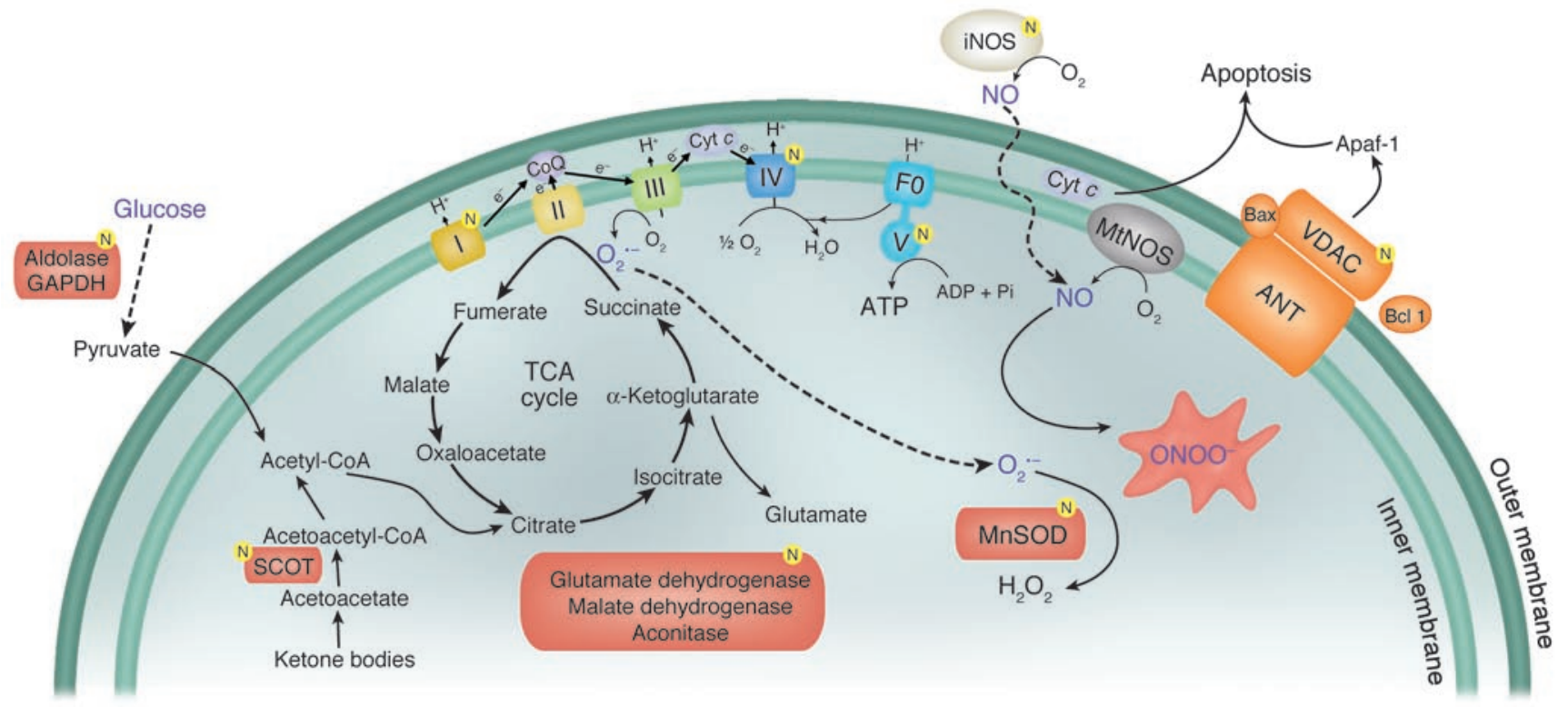

Figure 3

A diagrammatic representation of energy production in mitochondria and the mechanism of peroxynitrite generation. Excessive glycolysis and oxidative phosphorylation may result in more peroxynitrite generation in the critically ill. The ensuing nitration of mitochondrial complexes I and IV, MnSOD, GAPDH, and VDAC may suppress the activity of the mitochondrial electron transfer chain, impair detoxification of superoxide, shuttle glucose into toxic pathways, and increase apoptosis, respectively. These toxic effects may explain organ and cellular system failure related to adverse outcome in the critically ill. Proteins that are nitrated are indicated by the letter $\mathrm{N}$ in a yellow circle. Figure adapted with permission from American journal of physiology. Heart and circulatory physiology (44). TCA, tricarboxylic acid cycle; CoQ, coenzyme Q; Cyt c, cytochrome c; mtNOS, mitochondrial NO synthase; ANT, adenine nucleotide translocase; SCOT, succinyl-CoA:3-oxoacid CoA-transferase, ONOO-, peroxynitrite; F0, the portion of the mitochondrial ATP synthase that channels protons through the membrane.

these patients is not obvious. Analysis of liver and skeletal muscle biopsies, obtained immediately after death from nonsurvivors in the Leuven study, indicated that the classical insulin-regulated metabolic pathways in the liver did not respond to insulin $(35,36)$. For example, expression of IGFBP-1, normally under the inhibitory control of insulin, was unaltered by insulin in the critically ill (35). Circulating IGFBP-1 levels in both survivors and nonsurvivors were also unaltered by insulin at all times, and circulating IGFBP-1 levels correlated positively with the mRNA levels in the liver (35). Despite the fact that insulin did not effect IGFBP-1 in the critically ill patients, a high and rising serum IGFBP-1 concentration still predicted nonsurvival as early as 3 weeks prior to death. Furthermore, mRNA levels of phosphoenolpyruvate carboxykinase (PEPCK), the rate-limiting enzyme for hepatic gluconeogenesis, was unaffected by intensive insulin therapy (35). Together these findings may indicate that controlling gluconeogenesis was not the major factor responsible for the normalization of blood glucose levels with exogenous insulin in the critically ill. Expression of hepatic glucokinase, which controls glycogen synthesis, was also unaltered by intensive insulin therapy in critically ill patients, which, along with previous findings, confirms severe hepatic insulin resistance. In contrast, analysis of snap-frozen skeletal muscle biopsies of nonsurvivors in the Leuven study showed that skeletal muscle steady-state mRNA levels of the glucose transporter GLUT-4 and hexokinase II (HXK-II) were upregulated by insulin (36). Since GLUT-4 controls insulin-stimulated glucose uptake in muscle and since HXK-II is the rate-limiting step in intracellular insulin-stimulated glucose metabolism, the data suggest that in the critically ill patient, insulin lowers blood glucose predomi- nantly through increased skeletal muscle glucose uptake (36). However, true glucose kinetics can only be estimated from glucose turnover studies. Such a study, using a well-designed canine model of critical illness, recently confirmed a more severe insulin resistance in the liver as compared with other peripheral tissues (37). The transcription factor FOXO-1 is a forkhead protein expressed in liver and $\beta$ cells but not in adipose tissue or skeletal muscle (38). Dephosphorylated FOXO-1 translocates to the nucleus, where it stimulates transcription of PEPCK and glucose-6-phophatase. Insulin, via the PI3K/Akt branch of its signaling cascade, phosphorylates FOXO-1, whereby it is retained in the cytosol, blocking transcription of PEPCK or glucose-6-phosphatase. In the critically ill patient, adipose tissue and skeletal muscle remain relatively responsive to insulin, whereas the liver is much more resistant, and the $\beta$ cells appear unable to compensate fully for hyperglycemia. Although impaired phosphorylation of FOXO-1 could theoretically explain the difference among tissues, this possibility remains to be investigated.

\section{Mechanisms of accelerated glucose toxicity in the critically ill}

There are two possible explanations, not mutually exclusive, for hyperglycemia to be more acutely toxic in ICU patients than in healthy individuals or patients with diabetes mellitus. The first is accentuated cellular glucose overload, and the second is more pronounced toxic side effects of glycolysis and oxidative phosphorylation.

Cellular glucose overload in the critically ill. The central and peripheral nervous system, hepatocytes, and endothelial, epithelial, and immune cells are cellular compartments that take up glucose 
independently of insulin. Three glucose transporters, GLUT-1, GLUT-2, and GLUT-3, facilitate insulin-independent glucose transport in these tissues. In virtually all cell types, the GLUT-1 transporter ensures basal glucose uptake due to its low $K_{\mathrm{m}}(\approx 2$ $\mathrm{mM})$. In hepatocytes, renal tubular cells, pancreatic $\beta$ cells, and the gastrointestinal mucosa, the high $K_{\mathrm{m}}(\approx 66 \mathrm{mM})$ and high $V_{\max }$ of the GLUT-2 transporter allow glucose to enter the cell directly in equilibrium with the level of extracellular glucose. In neurons, GLUT-3 transporters $\left(K_{\mathrm{m}} \approx 9 \mathrm{mM}\right)$ are predominant. In normal cells, hyperglycemia downregulates GLUT-1 transporters, thereby protecting cells against glucose overload (32).

Cytokines, angiotensin II, endothelin-1, VEGF, TGF- $\beta$, and hypoxia have been shown to upregulate expression and membrane localization of GLUT-1 and GLUT-3 in different cell types, such as the endothelium, neurons, astrocytes, alveolar epithelial cells, and vascular smooth muscle cells (39-43). This "stress response" may overrule the normal protection of the cells against hyperglycemia, thus allowing cellular glucose overload. Hence, particularly in critical illness, characterized by high circulating levels of all these regulators, all organ systems that take up glucose passively may theoretically be at high risk for direct glucose toxicity. In contrast, skeletal muscle and the myocardium, which normally take up glucose predominantly via the insulin-dependent GLUT-4 transporter, may be relatively protected against toxic effects of circulating glucose.

More pronounced toxic side effects of oxidative phosphorylation in the critically ill. Besides cellular glucose overload, vulnerability to glucose toxicity may be due to increased generation of and/or deficient scavenging systems for ROS produced by activated glycolysis and oxidative phosphorylation (Figure 3). Glucose in the cytosol undergoes glycolysis, and its metabolite pyruvate is further transformed into acetyl-CoA, after which, in the presence of $\mathrm{O}_{2}$, oxidative phosphorylation generates ATP. Along with the generation of ATP by the mitochondrial respiratory chain complexes I-V, a small amount of superoxide is concomitantly produced. Normally, $2-5 \%$ of $\mathrm{O}_{2}$ used in the mitochondria is metabolized into superoxide, which is subsequently detoxified by manganese superoxide dismutase (MnSOD). When more glucose enters the cell and more pyruvate is being used for oxidative phosphorylation, more superoxide will be generated. Superoxide interacts with NO to form peroxynitrite, which nitrates proteins, such as mitochondrial complexes I and IV, MnSOD, GAPDH, and the voltage-dependent anion channel (VDAC). During critical illness as compared with the non-critically ill condition, more peroxynitrite may be generated due to cytokine-induced iNOS activation and hypoxia/ reperfusion-associated superoxide production (44). Hence, when cells of critically ill patients are overloaded with glucose, more superoxide and peroxynitrite production is to be expected. The ensuing nitration of mitochondrial complexes I and IV, MnSOD, GAPDH, and VDAC may theoretically suppress the activity of the mitochondrial electron transfer chain; impair detoxification of superoxide; shuttle glucose into toxic pathways - such as the polyol and hexosamine pathways - or induce advanced glycation end-product formation; and increase apoptosis, respectively.

Singer et al. showed that tyrosine nitration relates to suppressed activity of complex I in a cellular model of cytokine overload (45). Brownlee reported GAPDH inhibition linked to vascular damage of organs and tissues of patients with diabetes (46). We recently showed that preventing hyperglycemia with intensive insulin therapy beneficially affects the hepatocytic mitochondrial compartment of critically ill patients (47). Liver biopsies revealed pro- found ultrastructural abnormalities in hepatocytic mitochondria of patients to whom conventional insulin therapy was randomly allocated and thus with moderate hyperglycemia, whereas these abnormalities were virtually absent when normoglycemia was maintained during intensive care. The prevention or reversal of these morphological abnormalities had a functional correlate, as reflected by a higher activity of respiratory chain complex I and complex IV. In contrast to what was observed in the liver, electron microscopy revealed no major abnormalities in the mitochondria of skeletal muscle, and morphology as well as respiratory chain activity were not detectably affected by intensive insulin therapy in this tissue (47). The lack of effect on skeletal muscle mitochondria suggests a direct effect of glucose control, rather than of insulin, as the likely explanation. Whether these mitochondrial changes shown in critically ill patients are associated with alterations in protein nitration is currently being investigated.

Prevention of hyperglycemia-induced mitochondrial dysfunction in other cellular systems that allow glucose to enter passively, such as immune and endothelial cells, and in the central and peripheral nervous system would theoretically explain some of the protective effects of insulin therapy in critically ill patients. Increased blood glucose levels indeed have previously been shown to be associated with an increased risk of postoperative infection in patients with diabetes $(48,49)$. The Leuven study of nondiabetic ICU patients showed that maintaining normoglycemia with insulin prevents severe nosocomial infections and lethal sepsis (10). Polymorphonuclear neutrophil dysfunction (50), decreased intracellular bactericidal $(51,52)$ and opsonic activity $(50,53)$ following exposure to high concentrations of glucose, as well as nonenzymatic glycosylation of immunoglobulins (54) may play a role in the increased incidence of infections in patients with hyperglycemia. In an animal model of prolonged critical illness induced by trauma (55), it was recently shown that maintaining normoglycemia with insulin indeed rapidly affected innate immunity by preserving phagocytosis and oxidative burst function of monocytes (56). Ongoing studies will differentiate between the direct impact of preventing hyperglycemia and that of hyperinsulinemia on innate immunity.

Direct glucose toxicity may also explain why preventing moderate hyperglycemia with insulin clearly protects the central and the peripheral nervous system against secondary insults, as shown in the Leuven study $(10,34)$. Protection of the peripheral nervous system was evidenced by prevention of the development of critical illness polyneuropathy, which had immediate functional consequences such as shorter duration of mechanical ventilation and intensive care stay. Protection of the central nervous system of patients with isolated brain injury was revealed by the fact that intracranial pressure was lowered. Clinically, this finding coincided with fewer seizures, decreased incidence of diabetes insipidus, and improved long-term rehabilitation (34). Alternatively, a direct effect of insulin could have mediated some of these benefits, as insulin receptors are expressed at high levels in many areas of the brain and in different cell types (57). Indeed, local administration of insulin has shown to decrease uptake of $\gamma$-aminobutyric acid (GABA) by astrocytes and to increase the number and sensitivity of GABA receptors postsynaptically (58). This increased availability of GABA results in higher amplitude of miniature inhibitory postsynaptic currents. By inducing these GABA-mediated, neuroinhibitory effects, insulin may be directly neuroprotective. The reduced incidence of seizures observed in the Leuven study (34) corroborates such an explanation. 


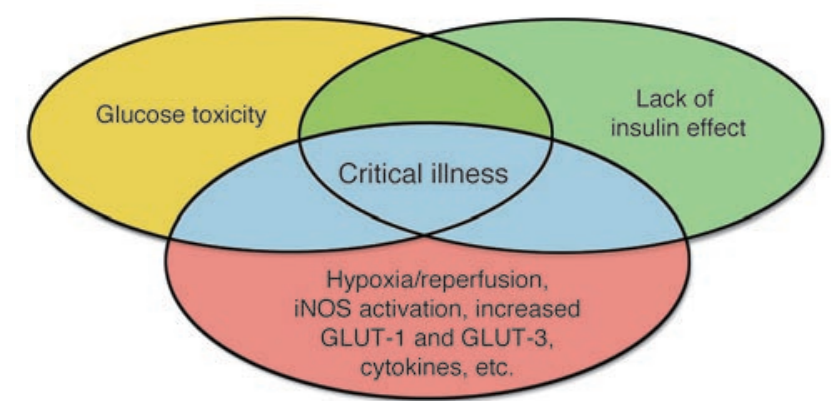

Figure 4

Venn diagram modeling the effect of the interaction between glucose toxicity and lack of insulin on the vulnerable state of critical illness. Complications of type 1 and type 2 diabetes are explained by hyperglycemia and/or lack of insulin effect. Critical illness is also characterized by hyperglycemia and lack of insulin effect, but additional risk factors render both of these effects more acutely toxic, as indicated by the blue shading. These risk factors include the post-hypoxia reperfused state, iNOS-activated NO generation, increased expression of GLUT-1 and GLUT-3 transporters, and cytokine-, neurological-, and hormoneinduced alterations in cellular processes. Hence, improved outcome of critical illness with insulin-titrated maintenance of normoglycemia is likely to be explained by the prevention of both direct glucose toxicity and insulin-induced effects that are independent of glucose control.

\section{Other metabolic effects of insulin in the critically ill}

Improvement of dyslipidemia. Insulin exerts other metabolic effects besides the control of blood glucose. As in patients with diabetes mellitus (59), abnormal serum lipid profiles are observed in critically ill patients (60-62). Most characteristically, circulating triglyceride levels are elevated, whereas the levels of HDL and LDL cholesterol are very low (63). On the other hand, the numbers of circulating small, dense LDL particles, which presumably are more proatherogenic than the medium and large LDL particles (64), are increased (65). Interestingly, this dyslipidemia could be partially restored by intensive insulin therapy, with almost complete reversal of the hypertriglyceridemia and a substantial increase in, but not normalization of, the serum levels of HDL and LDL (36). The roles of triglycerides in energy provision and of lipoproteins in coordinating transportation of lipid components (cholesterol, triglycerides, phospholipids, lipid-soluble vitamins) are well established (66). In addition, it has recently been shown that lipoproteins can scavenge endotoxins and by doing so are able to prevent death in animal models $(67,68)$. Multivariate logistic regression analysis demonstrated that the improvement of dyslipidemia with insulin in the Leuven study explained a significant part of the beneficial effect on mortality and organ failure and, surprisingly, its effects surpassed those of glycemic control and insulin dose (36). The latter was an important observation, because when controlling for all metabolic effects of insulin, including the lipid effect, the risk associated with a high dose of exogenously administered insulin $(32,69)$ disappeared (36). This finding negates the notion that high doses of insulin would be deleterious in the acute setting of critical illness (69). Rather, the data provide a strong argument in favor of titrating insulin to the doses that are required in order to achieve its metabolic effects. Evidently, blood glucose level is the easiest value to measure, and when insulin is titrated to normoglycemia, the other metabolic effects occur concomitantly. A molecular explanation for the dominant effect of serum lipid correction, however, still needs to be delineated.
Anabolic effects. In view of the catabolic state of critically ill patients with loss of lean body mass, despite adequate enteral or parenteral nutrition, the beneficial effects of insulin administration to the critically ill may have been mediated in part by its anabolic actions. Indeed, the binding of insulin to its receptor normally suppresses proteolysis and activates protein synthesis, both through the PI3K signaling pathway, and evokes cell proliferation through the MAPK pathway. Although in the Leuven study, no clinically obvious anabolic effects were observed, analysis of the skeletal muscle biopsies revealed a higher protein content with intensive insulin therapy (47). Also, in a rabbit model of prolonged critical illness, intensive insulin therapy appeared to prevent weight loss (56). Poor blood glucose control in patients with type 1 diabetes mellitus has been associated with low serum IGF-I levels, which can be increased by insulin therapy (70). Thus, the anabolic effects of insulin could be partly mediated by a rise in serum IGF-I. However, contrary to expectations, in the critically ill patients, serum IGF-I, acid-labile subunit, IGFBP-3, and GH-binding protein (GHBP) levels were further suppressed instead of increased by intensive insulin therapy, and circulating growth hormone (GH) levels were elevated instead of lowered (71). A molecular explanation for this transformation to a more "GH-resistant" state with insulin therapy in the critically ill is still lacking. Growth-promoting and anabolic effects of insulin are presumably mediated largely by its suppressive effect on IGFBP-1 $(72,73)$, whereby bioavailable IGF-I increases. Hence, the fact that intensive insulin therapy did not affect IGFBP-1 generation and serum levels in the critically ill patient may explain why such anabolic effects of insulin did not appear to play a major role in its beneficial effect on outcome.

\section{Other nonmetabolic effects of insulin in the critically ill}

Anti-inflammatory effects. Critical illness also resembles diabetes mellitus in the activation of the inflammatory cascade, although the inflammation, as reflected by a high circulating level of C-reactive protein (CRP), in the critically ill is several times more pronounced than in diabetic patients. Intensive insulin therapy prevented excessive inflammation in critically ill patients, as indicated by lowered serum CRP and mannose-binding lectin levels $(10,74)$. The antiinflammatory effects of intensive insulin therapy were present independently of its preventive effect on infections. This finding was recently confirmed in an experimental rabbit model of prolonged critical illness (56). The exact mechanisms explaining the anti-inflammatory effects of intensive insulin therapy have not yet been unraveled. Insulin may exert direct anti-inflammatory effects through its suppression of NF- $\mathrm{BB}$-regulated pathways, including the production of inflammatory cytokines such as TNF- $\alpha$, macrophage migration-inhibitory factor, and the generation of superoxide $(75,76)$. Alternatively, prevention of hyperglycemia may also play a role. The effect of insulin-titrated maintenance of normoglycemia on inflammation in the critically ill (74) was no longer independently related to the outcome benefit when the changes in lipid metabolism were taken into account (36). This observation is suggestive of a link between the anti-inflammatory effect of intensive insulin therapy and its amelioration of the lipid profile.

Preventing endothelial dysfunction and bypercoagulation. Diabetes mellitus and critical illness are both hypercoagulable states (77, $78)$. In the critically ill, this may contribute to the risk of organ failure. Putative causes in diabetes include vascular endothelium dysfunction (79), increased circulating levels of several clotting factors $(80,81)$, elevated platelet activation $(82,83)$, and inhibition 
of the fibrinolytic system (84). Levels of the anticoagulant protein $\mathrm{C}$ are also decreased (85). The similarities between critical illness and diabetes $(86,87)$; the powerful preventive effect of intensive insulin therapy on septicemia, multiple organ failure, and mortality (10); the influence of intensive insulin therapy on endothelial activation; and the balance between coagulation and fibrinolysis in the critically ill should be investigated in detail. On the one hand, insulin has been shown to activate $\mathrm{Ca}^{2+}$-independent eNOS generation in endothelial cells (88). On the other hand, the recent concept of "selective insulin resistance," involving unresponsiveness of the PI3K post-receptor signaling pathway but responsiveness of the Ras-MAP kinase pathways in endothelial cells, raises the possibility of insulin-induced aggravation of endothelial activation, the latter evoked by hyperglycemia, angiotensin II, and vascular growth factors such as VEGF. Since VEGF is known to be highly expressed in the inflammatory phase of critical illness, this hypothesis was recently tested in samples obtained in the Leuven study. In line with the clinical benefits observed, circulating levels of the adhesion molecules soluble ICAM-I and E-selectin were downregulated by intensive insulin therapy (G. Van den Berghe, unpublished data). This suggests that in the critically ill, the PI3K pathway in the endothelium, as in skeletal muscle, remains at least partially responsive, in contrast to the metabolic pathways in the liver, which are highly insulin resistant.

Anti-apoptotic effects. More than 4 decades ago the concept was introduced that glucose, insulin, and potassium (GIK), administered concomitantly, could protect the ischemic myocardium (89). Opie suggested that the mechanism behind this cardioprotective effect of GIK is ATP generation when $\mathrm{O}_{2}$ supply is limited by promoting glycolysis instead of FFA oxidation (90). Currently, experimental data support the hypothesis that insulin itself has direct cardioprotective effects during reperfusion, mainly via anti-apoptotic properties that are independent of glucose uptake (91-93). The insulin signaling pathways involved include PI3K, Akt, and eNOS phosphorylation (92). Whether such direct insulininduced cell survival plays a role in mediating the observed protection of organ function in the critically ill remains unclear.

\section{Future directions}

It is clear that several mechanisms are involved and interrelated in explaining the clinical benefits of intensive insulin therapy in the critically ill (Figure 4). Accelerated toxicity of hyperglycemia and lack of insulin effect during critical illness explain why the consequences are so rapidly deleterious in this condition. The direct effects of preventing hyperglycemia as well as the distinct metabolic and nonmetabolic effects of insulin that occur concomitantly with the glycemic control are likely to play a role. The relative contribution of those different mechanisms, however, is presently unknown. Furthermore, several questions await an answer. The exact molecular basis for the increased susceptibility for these toxic effects in the critically ill patient remains to be explored. It also remains unclear which of the insulin signal transduction pathways in different target tissues respond to insulin treatment and how these effects mediate the protection on organ function and overall outcome. Various hypotheses have been advanced, and these can be tested by analysis of tissue samples obtained from patients and from experimental animals in carefully designed studies. Further investigation of the mitochondrial abnormalities in tissues other than the liver and skeletal muscle, such as neurons, kidneys, and endothelial and epithelial cells, as well as the selective impact of insulin and glucose control on this cellular compartment should be investigated in great detail. Furthermore, exploring the molecular link between improved glycemic and lipid control and insulin, endothelial function, inflammation, and clinical outcome will be required. Finally, manipulation of those pathways that are linked with outcome but appear unresponsive to intensive insulin therapy may point to the potential for other therapeutic strategies to further improve survival in the ICU.

\section{Conclusion}

The results of the clinical study performed in Leuven demonstrated that a simple metabolic intervention, maintaining normoglycemia with insulin, improved survival and reduced morbidity of critically ill patients. This reflected major clinical progress in the modern era of intensive care. Furthermore, this clinical study has opened a whole new area of basic research besides the ongoing search for additional clinical applications. Indeed, only some of the underlying mechanisms have been studied, and many more pathways need to be investigated in great detail. Results from fundamental research carefully designed to elucidate how the clinical benefits were brought about are likely to set off development of new strategies for further improving outcome of critically ill patients and perhaps also of other target populations within and outside the hospital setting.

\section{Acknowledgments}

I thank Roger Bouillon, Ilse Vanhorebeek, and Lies Langouche for critically reviewing the manuscript. This work was supported by the Fund for Scientific Research, Flanders, Belgium (G.0278.03), the Research Council of the Catholic University of Leuven (OT 03/56), and the Belgian Foundation for Research in Congenital Heart Diseases. The author is a Fundamental Clinical Research Investigator (G.3C05.95N) for the Fund for Scientific Research, Flanders, Belgium, and holds an unrestrictive Catholic University of Leuven - Novo Nordisk Chair of Research.

Address correspondence to: Greet Van den Berghe, Department of Intensive Care Medicine, Catholic University of Leuven, B-3000 Leuven, Belgium. Phone: 32-16-34-40-21; Fax: 32-16-34-40-15; E-mail: greta.vandenberghe@med.kuleuven.ac.be.
1. Lassen, H.C.A. 1953. A preliminary report on the 1952 epidemic of poliomyelitis in Copenhagen with special reference to the treatment of respiratory insufficiency. Lancet. i:37-40.

2. Fink, M.P., and Evans, T.W. 2002. Mechanisms of organ dysfunction in critical illness: report from a round table conference held in Brussels. Intensive Care Med. 28:369-375.

3. Fink, M.P. 2001. Cytopathic hypoxia. Mitochondrial dysfunction as mechanism contributing to organ dysfunction in sepsis. Crit. Care Clin. 17:219-237.

4. Brealey, D., et al. 2002. Association between mitochondrial dysfunction and severity and outcome of septic shock. Lancet. 360:219-223.

5. Singer, M., and Brealey, D. 1999. Mitochondrial dysfuntion in sepsis. Biochem. Soc. Symp. 66:149-166.

6. Crouser, E.D., Julian, M.W., Blaho, D.V., and Pfeiffer, D.R. 2002. Endotoxin-induced mitochondrial damage correlates with impaired respiratory activity. Crit. Care Med. 30:276-284.

7. Amato, M.B., et al. 1998. Effect of a protective-ventilation strategy on mortality in the acute respiratory distress syndrome. N. Engl. J. Med. 338:347-354.

8. Rivers, E., et al. 2001. Early goal-directed therapy in the treatment of severe sepsis and septic shock. N. Engl. J. Med. 345:1368-1377.

9. Bernard, G.R., et al. 2001. Efficacy and safety of recombinant human activated protein $\mathrm{C}$ for severe sepsis. N. Engl. J. Med. 344:699-709.

10. Van den Berghe, G., et al. 2001. Intensive insulin therapy in critically ill patients. N. Engl. J. Med. 345:1359-1367.

11. Bernard, C. 1878. Leçons sur les phénomènes de la vie communs aux animaux et aux végétaux. Volume 1. J.B. Baillière et Fils. Paris, France. 564 pp.

12. Thorell, A., Nygren, J., and Ljungqvist, O. 1999. 
Insulin resistance: a marker of surgical stress. Curr Opin. Clin. Nutr. Metab. Care. 21:69-78.

13. McCowen, K.C., Malhotra, A., and Bistrian, B.R. 2001. Stress-induced hyperglycaemia. Crit. Care Clin. 17:107-124.

14. Van den Berghe, G., et al. 1999. Reactivation of pituitary hormone release and metabolic improvement by infusion of growth hormone-releasing peptide and thyrotropin-releasing hormone in patients with protracted critical illness. J. Clin. Endocrinol. Metab. 84:1311-1323.

15. Van den Berghe, G., et al. 2000. A paradoxical gender dissociation within the growth hormone/insulin-like growth factor I axis during protracted critical illness. J. Clin. Endocrinol. Metab. 85:183-192.

16. Wolfe, R.R., Durkot, M.J., Allsop, J.R., and Burke, J.F. 1979. Glucose metabolism in severely burned patients. Metabolism. 28:210-220.

17. Wolfe, R.R., Herndon, D.N., Jahoor, F., Miyoshi, H., and Wolfe, M. 1987. Effects of severe burn injury on substrate cycling by glucose and fatty acids. N. Engl. J. Med. 317:403-408.

18. Mizock, B.A. 1995. Alterations in carbohydrate metabolism during stress: a review of the literature. Am. J. Med. 98:75-84.

19. Grimble, R.F. 2002. Inflammatory status and insulin resistance. Curr. Opin. Clin. Nutr. Metab. Care. 5:551-559.

20. Marette, A. 2002. Mediators of cytokine-induced insulin resistance in obesity and other inflammatory settings. Curr. Opin. Clin. Nutr. Metab. Care. 5:377-383.

21. Senn, J.J., et al. 2003. Suppressor of cytokine signaling-3 (SOCS-3), a potential mediator of interleukindependent insulin resistance in hepatocytes. J. Biol. Chem. 278:13740-13746.

22. Rui, L., Yuan, M., Frantz, D., Shoelson, S., and White, M.F. 2002. SOCS-1 and SOCS-3 block insulin signaling by ubiquitin-mediated degradation of IRS1 and IRS2. J. Biol. Chem. 277:42394-42398.

23. McCowen, K.C., et al. 2001. Sustained endotoxemia leads to marked down-regulation of early steps in the insulin-signaling cascade. Crit. Care Med. 29:839-846.

24. Jiang, Z.Y., et al. 1999. Characterization of selective resistance to insulin signaling in the vasculature of obese Zucker (fa/fa) rats. J. Clin. Invest. 104:447-457.

25. Cusi, K., et al. 2000. Insulin resistance differentially affects the PI 3-kinase-and MAP kinase-mediated signaling in human muscle. J. Clin. Invest. 105:311-320.

26. Draznin, B., et al. 2000. Effects of insulin on prenylation as a mechanism of potential detrimental influence of hyperinsulinemia. Endocrinology. 141:1310-1316.

27. Golovchenko, I., Goalstone, M.L., Watson, P., Brownlee, M., and Draznin, B. 2000. Hyperinsulinemia enhances transcriptional activity of Nuclear Factor$\mathrm{kB}$ induced by angiotensin II, hyperglycemia, and advanced glycosylation end products in vascular smooth muscle cells. Circ. Res. 87:746-752.

28. Montagnani, M., et al. 2002. Inhibition of phosphatidylinositol 3-kinase enhances mitogenic actions of insulin in endothelial cells. J. Biol. Chem. 277:1794-1799.

29. Mizock, B.A. 2001. Alterations in fuel metabolism in critical illness: hyperglycaemia. Best Pract. Res Clin. Endocrinol. Metab. 15:533-551.

30. Malmberg, K., et al. 1995. Randomized trial of insulin-glucose infusion followed by subcutaneous insulin treatment in diabetic patients with acute myocardial infarction (DIGAMI study): effects on mortality at 1 year. J. Am. Coll. Cardiol. 26:57-65.

31. Krinsley, J.S. 2004. Effect of an intensive glucose management protocol on the mortality of critically ill adult patients. Mayo Clin. Proc. 79:992-1000.

32. Klip, A., Tsakiridis, T., Marette, A., and Ortiz, P.A. 1994. Regulation of expression of glucose trans- porters by glucose: a review of studies in vivo and in cell cultures. FASEB J. 8:43-53.

33. Van den Berghe. G., et al. 2003. Outcome benefit of intensive insulin therapy in the critically ill: insulin dose versus glycemic control. Crit. Care Med. 31:359-366.

34. Van den Berghe, G., Schoonheydt, K., Becx, P., Bruyninckx, F., and Wouters, P.J. 2004. Intensive insulin therapy protects the central and peripheral nervous system of intensive care patients. In: Program and abstracts of the 86th Annual Meeting of the Endocrine Society. New Orleans, Louisiana, USA. June 16-19, 2004. Abstract OR8-2, p. 79.

35. Mesotten, D., et al. 2002. Regulation of insulinlike growth factor binding protein-1 during protracted critical illness. J. Clin. Endocrinol. Metab. 87:5516-5523.

36. Mesotten, D., Swinnen, J., Vanderhoydonc, F., Wouters, P.J., and Van den Berghe, G. 2004. Contribution of circulating lipids to the improved outcome of critical illness by glycemic control with intensive insulin therapy. J. Clin. Endocrinol. Metab. 89:219-226.

37. Donmoyer, C.M., et al. 2003. Infection impairs insulin-dependent hepatic glucose uptake during total parenteral nutrition. Am. J. Physiol. Endocrinol. Metab. 284:E574-E582.

38. Puigserver, P., et al. 2003. Insulin-regulated hepatic gluconeogenesis through FOXO1-PGC-1alpha interaction. Nature. 423:550-555.

39. Pekala, P., Marlow, M., Heuvelman, D., and Connolly, D. 1990. Regulation of hexose transport in aortic endothelial cells by vascular permeability factor and tumor necrosis factor-alpha, but not by insulin. J. Biol. Chem. 265:18051-18054.

40. Shikhman, A.R., Brinson, D.C., Valbracht, J., and Lotz, M.K. 2001. Cytokine regulation of facilitated glucose transport in human articular chondrocytes. J. Immunol. 167:7001-7008.

41. Quinn, L.A., and McCumbee, W.D. 1998. Regulation of glucose transport by angiotensin II and glucose in cultured vascular smooth muscle cells. J. Cell. Physiol. 177:94-102.

42. Clerici, C., and Matthay, M.A. 2000. Hypoxia regulates gene expression of alveolar epithelial transport proteins. J. Appl. Physiol. 88:1890-1896.

43. Sanchez-Alvarez, R., Tabernero, A., and Medina, J.M. 2004. Endothelin-1 stimulates the translocation and upregulation of both glucose transporter and hexokinase in astrocytes: relationship with gap junctional communication. J. Neurochem. 89:703-714.

44. Aulak, K.S., Koeck, T., Crabb, J.W., and Stuehr, D.J. 2004. Dynamics of protein nitration in cells and mitochondria. Am. J. Physiol. Heart Circ. Physiol. 286:H30-H38.

45. Frost, M., Wang, Q., Moncada, S., and Singer, M. 2004. Bi-phasic, oxygen- and NO-dependent modulation of complex I activity in activated macrophages by S-nitrosylation and nitration. Am. J. Physiol. In press.

46. Brownlee, M. 2001. Biochemistry and molecular cell biology of diabetic complications. Nature. 414:813-820.

47. Vanhorebeek, I., et al. 2004. Strict blood glucose control with insulin in critically ill patients protects hepatocytic mitochondrial ultrastructure and function. Lancet. In press.

48. Pozzilli, P., and Leslie, R.D. 1994. Infections and diabetes: mechanisms and prospects for prevention. Diabet. Med. 11:935-941.

49. Funari, A.P., Zerr, K.J., Grunkemeier, G.L., and Starr, A. 1999. Continuous intravenous insulin infusion reduces the incidence of deep sternal wound infection in diabetic patients after cardiac surgical procedures. Ann. Thorac. Surg. 67:352-360.

50. Rassias, A.J., et al. 1999. Insulin infusion improves neutrophil function in diabetic cardiac surgery patients. Anesth. Analg. 88:1011-1016.
51. Nielson, C.P., and Hindson, D.A. 1989. Inhibition of polymorphonuclear leukocyte respiratory burst by elevated glucose concentrations in vitro. Diabetes. 38:1031-1035.

52. Perner, A., Nielsen, S.E., and Rask-Madsen, J. 2003. High glucose impairs superoxide production from isolated blood neutrophils. Intensive Care Med. 29:642-645.

53. Rayfield, E.J., et al. 1982. Infection and diabetes: the case for glucose control. Am. J. Med. 72:439-450.

54. Black, C.T., Hennessey, P.J., and Andrassy, R.J. 1990. Short-term hyperglycemia depresses immunity through nonenzymatic glycosylation of circulating immunoglobulin. J. Trauma. 30:830-832.

55. Weekers, $\mathrm{F}$. et al. 2002. A novel in vivo rabbit model of hypercatabolic critical illness reveals a bi-phasic neuroendocrine stress response. Endocrinology. 143:764-774

56. Weekers, F., et al. 2003. Metabolic, endocrine and immune effects of stress hyperglycemia in a rabbit model of prolonged critical illness. Endocrinology. 144:5329-5338

57. Bruning, J.C., et al. 2000. Role of brain insulin receptor in control of body weight and reproduction. Science. 289:2122-2155.

58. Vincent, A.M., Brownlee, M., and Russell, J.W. 2002. Oxidative stress and programmed cell death in diabetic neuropathy. Ann. N. Y. Acad. Sci. 959:368-383.

59. Taskinen, M.R. 2001. Pathogenesis of dyslipidemia in type 2 diabetes. Exp. Clin. Endocrinol. Diabetes. 109:S180-S188.

60. Lanza-Jacoby, S., Wong, S.H., Tabares, A., Baer, D., and Schneider, T. 1992. Disturbances in the composition of plasma lipoproteins during gramnegative sepsis in the rat. Biochim. Biophys. Acta. 1124:233-240.

61. Khovidhunkit, W., Memon, R.A., Feingold, K.R., and Grunfeld, C. 2000. Infection and inflammation-induced proatherogenic changes of lipoproteins [review]. J. Infect. Dis. 181:S462-S472.

62. Carpentier, Y.A., and Scruel, O. 2002. Changes in the concentration and composition of plasma lipoproteins during the acute phase response. Curr. Opin. Clin. Nutr. Metab. Care. 5:153-158.

63. Gordon, B.R., et al. 1996. Low lipid concentrations in critical illness: implications for preventing and treating endotoxemia. Crit. Care Med. 24:584-589.

64. Kwiterovich, P.O. 2002. Lipoprotein heterogeneity: diagnostic and therapeutic implications. Am. J. Cardiol. 90:1i-10i.

65. Feingold, K.R., et al. 1993. The hypertriglyceridemia of acquired immunodeficiency syndrome is associated with an increased prevalence of low density lipoprotein subclass pattern B. J. Clin. Endocrinol. Metab. 76:1423-1427.

66. Tulenko, T.N., and Sumner, A.E. 2002. The physiology of lipoproteins. J. Nucl. Cardiol. 9:638-649.

67. Harris, H.W., Grunfeld, C., Feingold, K.R., and Rapp, J.H. 1990. Human very low density lipoproteins and chylomicrons can protect against endotoxin-induced death in mice. J. Clin. Invest. 86:696-702.

68. Harris, H.W., et al. 1993. Chylomicrons alter the fate of endotoxin, decreasing tumor necrosis factor release and preventing death. J. Clin. Invest. 91:1028-1034.

69. Finney, S.J., Zekveld, C., Elia, A., and Evans, T.W. 2003. Glucose control and mortality in critically ill patients. JAMA. 290:2041-2047.

70. Brismar, K., Fernqvist-Forbes, E., Wahren, J., and Hall, K. 1994. Effect of insulin on the hepatic production of insulin-like growth factor-binding protein-1 (IGFBP-1), IGFBP-3, and IGF-I in insulin-dependent diabetes. J. Clin. Endocrinol. Metab. 79:872-878.

71. Mesotten, D., et al. 2004. Regulation of the somatotropic axis by intensive insulin therapy during protracted critical illness. J. Clin. Endocrinol. Metab. 
89:3105-3113.

72. Suikkari, A.M., et al. 1988. Insulin regulates the serum levels of low molecular weight insulin-like growth factor-binding protein. J. Clin. Endocrinol. Metab. 66:266-272.

73. Suwanichkul, A., Morris, S.L., and Powell, D.R. 1993. Identification of an insulin-responsive element in the promoter of the human gene for insulin-like growth factor binding protein-1. J. Biol. Chem. 268:17063-17068.

74. Hansen, T.K., Thiel, S., Wouters, P.J., Christiansen, J.S., and Van den Berghe, G. 2003. Intensive insulin therapy exerts antiinflammatory effects in critically ill patients and counteracts the adverse effect of low mannose-binding lectin levels. J. Clin. Endocrinol. Metab. 88:1082-1088.

75. Das, U.N. 2001. Is insulin an antiinflammatory molecule? Nutrition. 17:409-413.

76. Dandona, P., et al. 2001. Insulin inhibits intranuclear factor kappaB and stimulates IkappaB in mononuclear cells in obese subjects: evidence for an anti-inflammatory effect? J. Clin. Endocrinol. Metab. 86:3257-3265.

77. Carr, M.E. 2001. Diabetes mellitus: a hypercoagulable state. J. Diabetes Complicat. 15:44-54.

78. Calles-Escandon, J., Garcia-Rubi, E., Mirza, S., and Mortensen, A. 1999. Type 2 diabetes: one disease, multiple cardiovascular risk factors. Coron. Artery Dis. 10:23-30.
79. Williams, E., Timperley, W.R., Ward, J.D., and Duckworth, T. 1980. Electron microscopical studies of vessels in diabetic peripheral neuropathy. J. Clin. Pathol. 33:462-470.

80. Patrassi, G.M., Vettor, R., Padovan, D., and Girolami, A. 1982. Contact phase of blood coagulation in diabetes mellitus. Eur. J. Clin. Invest. 12:307-311.

81. Carmassi, F., et al. 1992. Coagulation and fibrinolytic system impairment in insulin dependent diabetes mellitus. Thromb. Res. 67:643-654.

82. Hughes, A., et al. 1983. Diabetes, a hypercoagulable state? Hemostatic variables in newly diagnosed type 2 diabetic patients. Acta Haematol. 69:254-259.

83. Garcia Frade, L.J., et al. 1987. Diabetes mellitus as a hypercoagulable state: its relationship with fibrin fragments and vascular damage. Thromb. Res. 47:533-540.

84. Carmassi, F., et al. 1992. Coagulation and fibrinolytic system impairment in insulin dependent diabetes mellitus. Thromb. Res. 67:643-654.

85. Vukovich, T.C., and Schernthaner, G. 1986 Decreased protein C levels in patients with insulin-dependent type I diabetes mellitus. Diabetes. 35:617-619.

86. Garcia Frade, L.J., et al. 1987. Changes in fibrinolysis in the intensive care patient. Thromb. Res. 47:593-599.

87. Mavrommatis, A.C., et al. 2001. Activation of the fibrinolytic system and utilization of the coagulation inhibitors in sepsis: comparison with severe sepsis and septic shock. Intensive Care Med. 27:1853-1859.

88. Montagnani, M., Chen, H., Barr, V.A., and Quon, M.J. 2001. Insulin-stimulated activation of e-NOS is independent of Ca2+ but requires phosphorylation by Akt at Ser(1179). J. Biol. Chem. 276:30392-30398.

89. Sodi-Pallares, D., Testelli, M.R., and Fishleder, B. 1962. Effects of an intravenous infusion of a potassium-insulin-glucose solution on the electrocardiographic signs of myocardial infarction. A preliminary clinical report. Am. J. Cardiol. 9:166-181.

90. Opie, L. 1970. The glucose hypothesis: relation to acute myocardial ischemia. J. Mol. Cell. Cardiol. 1:107-114.

91. Jonassen, A., Aasum, E., Riemersma, R., Mjos, O., and Larsen, T. 2000. Glucose-insulin-potassium reduces infarct size when administered during reperfusion. Cardiovasc. Drugs Ther. 14:615-623.

92. Gao, F., et al. 2002. Nitric oxide mediates the antiapoptotic effect of insulin in myocardial ischemiareperfusion: the role of PI3-kinase, Akt, and eNOS phosphorylation. Circulation. 105:1497-1502.

93. Jonassen, A., Sack, M., Mjos, O., and Yellon, D. 2001. Myocardial protection by insulin at reperfusion requires early administration and is mediated via Akt and p70s6 kinase cell-survival signalling. Circ. Res. 89:1191-1198. 